\title{
The placebo enigma revisited
}

\author{
JMS Pearce
}

The word placebo means: I shall please. A multifaceted reaction, it depends on interactions between the doctor, treatment, and patient. ${ }^{1}$ This condensed essay considers the rival organicfunctional conceptions, the nature of placebos, their mechanisms, and their use in therapeutic trials.

\section{History and definition}

The medical word 'placebo' dates from the late 18th century. In 1811, Quincy's Lexicon-Medicum defined it as 'an epithet given to any medicine adapted more to please than to benefit the patient.'2 In 1807, Thomas Jefferson, recording what he called the pious fraud, noted a successful physician who claimed he used more 'bread pills, drops of colored water, and powders of hickory ashes, than of all other medicines put together'. In an era when pharmacologically effective medicines were few, most physicians did not consider the bread pill as a deception or threat to ethical integrity. In a 1930s study, Evans and Hoyle used sodium bicarbonate to treat angina with $38 \%$ improvement, comparable to nitroglycerin. ${ }^{3}$ Similar studies show placebo response in one third of the population, but the proportion depends on:

- study design

- the illness

- the subject under investigation

- the doctor or therapist (Fig 1).

\section{Recent definitions}

The placebo may be any kind of intervention without intrinsic therapeutic effect. Recent definitions include:

The placebo response is an improvement of subjective and objective outcomes while taking an inert substance or undergoing a sham procedure. ${ }^{4}$

A drug, medicine, therapy, etc, prescribed more for the psychological benefit to the patient of being given treatment than for any direct physiological effect; esp. one with no specific therapeutic effect on a patient's condition, but believed by the patient to be therapeutic (and sometimes therefore effective). Also: a substance with no therapeutic effect used as a control in testing new drugs, etc.; a blank sample in a test.

Oxford English Dictionary

But some deny placebo reactions. ${ }^{5}$

JMS Pearce, emeritus consultant neurologist, Department of Neurology, Hull Royal Infirmary

\section{Placebo myths}

Placebos provoke controversy. As Patrick Wall (1925-2001) remarked:

It is an unpopular topic because it is confused with quackery or seen as an expensive artefact or taken to challenge the rationale of a therapy or to mock the reality of the senses. In order to avoid taking the subject seriously, myths are invented claiming that placebos work only on hysterics or hallucinators or that they are the equivalent of doing nothing or that they act only on the mental results of pain and not on the pain itself.

He outlined several placebo myths:

- 'There is nothing wrong with placebo responders in the first place.'

- 'A fixed per cent of people (usually around 30\%) are placebo responders.'

- 'Only those open to suggestion respond to placebos...The real message [in this myth] is that placebo responders aren't real people, They must be peculiar, not like you and me.'

- 'Giving a patient a placebo is the equivalent of doing nothing - the disease or injury is simply living out its natural history...a placebo does something positive on top of that natural history.'

- 'Only small pains respond to a placebo.'

In practice, placebo reaction is commonly affected by interventions ranging from history-taking and reassurance to surgery. But many still erroneously regard the placebo reaction

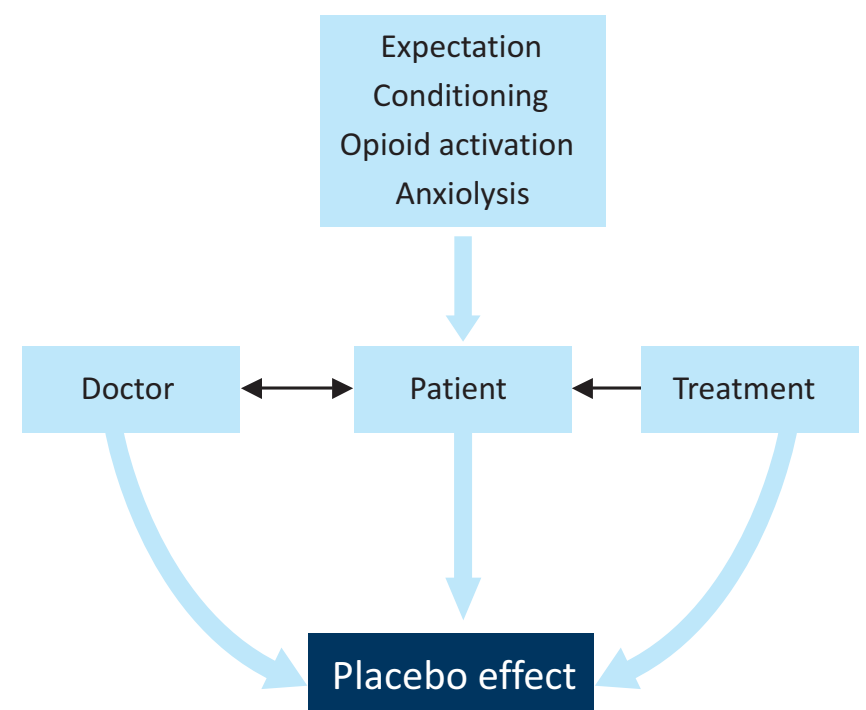

Fig 1. Simplified schema: placebo effect. 
as a means of separating functional from organic states, ${ }^{1}$ ignoring the objective changes attendant on using placebos (cf. THE Oxford English Dictionary definition).

\section{Placebo-controlled trials}

The placebo, a 'humble humbug', acquired scientific value in controlled trials. Henry Beecher reviewed 15 placebo-controlled trials and found a placebo effect of $35.2 \% .^{7}$

\section{The clinical spectrum of placebo reaction}

Placebos affect not only pain and other symptoms, but objective signs of pathology and disordered physiology. Patients with any distressing complaint may show placebo responses. Yet some deny the effect. A meta-analysis of 114 studies failed to find any significant difference between patients receiving no treatment and those receiving placebo. ${ }^{5}$ However, the authors disingenuously tried to subtract psychological and interpersonal therapeutic effects from placebo responses. A substantial placebo effect occurs in depression, fatigue, ${ }^{8}$ and severe pain. In postoperative pain, one third of patients obtain substantial analgesia from inert substances: 'Consequently the only thing learned from a placebo trial is whether or not the patient is placebo-positive. The trial is of no assistance in separating psychogenic from organic pain. ${ }^{9}$ Often neither doctor nor patient is aware of the placebo effect in a given treatment and although circadian patterns of placebo reactions are observed, ${ }^{10}$ it is not know whether all, or only some patients, show a placebo reaction at any time.

\section{Which treatments act as placebos?}

Placebo effects have been shown in drugs, homeopathic medicines, acupuncture, psychotherapy, transcutaneous electrical nerve stimulation and surgery. In a classic double-blind trial of internal mammary artery ligation and a sham operation for angina, many sham operations resulted in improved angina, exercise tolerance and electrocardiograms. ${ }^{11}$

Placebo action and efficacy depend on clinical presentation and circumstances. Certain coloured oral preparations have more antidepressant effects than other colours of the same medication. ${ }^{12}$ Injections may be more effective than oral use of the same drug. A physician's personality, dress, demeanour, voice and body language may all contribute to the effect. ${ }^{13}$ Perceived clinical interest, caring and sympathy in the attending physician similarly have an important, if undefined, role in symptom relief.

\section{Which symptoms respond to placebo?}

Placebo relief is well recorded in most painful syndromes, including migraine, ${ }^{14}$ backache, dysmenorrhoea, irritable bowel syndrome. Depression, asthma, hypertension, congestive cardiac failure, tardive dyskinesia and Parkinsonism can also respond to placebo. Parkinsonians respond to placebo by decreased neuronal firing of the subthalamic nucleus and substantia nigra, and increased firing in thalamic nuclei. ${ }^{15}$ Antibody responses can also be influenced.

In a double-blind study in 150 patients following removal of impacted molars, facial swelling, trismus, and serum C-reactive protein were reduced both in ultrasound-treated and 'mock' ultrasound groups. ${ }^{16}$ Thus objective signs can be modified by placebo.

\section{Placebo characteristics}

Interestingly, placebo medications exhibit many characteristics of conventional drugs. These include time-effect curves, peak, cumulative, and carry-over effects, and a wide spectrum of sideeffects (nocebo effects). Placebo administration in 109 placebocontrolled studies showed side effects in 19\% during placebo administration. ${ }^{17}$

\section{Placebos in clinical trials}

Few phenomena are as misunderstood as the placebo trial. ${ }^{9}$ The duration of placebo responses vary widely. These factors relate to the symptom treated, the doctor's predictions and the effects anticipated by the patient. Interpretation is often confounded by spontaneous remission. Thus it is dangerous to infer that apparent benefit is caused by either active drug, or placebo. Patients may try to please the doctor and spuriously report improvement. ${ }^{5}$ The attention and inclusion in a trial increase the effect.

However, reliable evaluation of treatments would be difficult without placebos. No other type of control groups can adduce findings as compelling and interpretable as a statistically significant drug-placebo difference. ${ }^{18}$

\section{Mechanisms}

There are four hypotheses:

- Endorphin release. Emotional factors can increase or decrease pain perception. The administration of naltrexone before dental surgery reduced the analgesic response to both placebo and codeine: a hyperalgesic effect. But the mechanism whereby opioid, anti-opioid and B-lipotrophin neuropeptides cause placebo-induced analgesia is uncertain. Using PET and the $\mu$-opioid selective radiotracer $[(11) \mathrm{C}]$ carfentanil placebo-induced activation of opioid neurotransmission in several brain regions has been demonstrated. ${ }^{19}$ However, these mechanisms do not explain nonpainful symptoms alleviated by placebos.

- Anxiolytic. It is often wrongly assumed that response to placebo implies psychogenesis. Depression increases the perception of pain. Both tricyclic and 5-HT reuptake inhibitors can produce analgesia in neuropathic pain. Physiological neural changes ${ }^{20}$ may be mediated by frontal cortical areas that relate to cognitive expectations. Placebo benefits also involve neurophysiological or neurochemical changes and side-effects, closely allied to conventional drug therapy. However, the mechanisms of placebo analgesia 
remain uncertain. In the notion of cognitive versus somatic pain, somatic pain is related to the source of nociception, while the patient's awareness and cognition determine the perceived pain. This separation of somatic and psychological substrates is as artificial as it is simplistic.

- Expectation. The placebo reactor's expectation of success is postulated as an underlying mechanism. Quelling anxiety, reassurance, explanation, all contribute to therapeutic success. The physician's actions are expected to provide relief. Since expectation also involves the attitudes (facial, gestural and spoken) and communication by doctors and nurses, this situation is difficult of analysis, but a potent factor in all treatments.

- Conditioning. Voudouris and colleagues have shown that in subjects in whom the threshold to a painful current had been established, an inert ointment said to have anaesthetic properties, reduced pain. A second group given the same suggestion and ointment had the painful current secretly reduced. This second group later became strong placebo responders to the originally painful current and ointment. $^{21}$ Another investigation of blood pressure responses to atenolol and placebo confirmed the effects of conditioning.

\section{Ethics of placebo trials ${ }^{22}$}

Both the scientific and ethical status of placebos provoke controversy. Respected opinions warn that a placebo-controlled trial is ethical only when there is no known effective treatment available. Sir Austin Bradford Hill commented:

\section{Is it ethical to use a placebo? The answer to this question will depend, I suggest, upon whether there is already available an orthodox treatment of proved or accepted value. If there is such an orthodox treatment the question will hardly arise, for the doctor will wish to know whether a new treatment is more, or less, effective than the old, not that it is more effective than nothing.}

A pragmatic view is that medicine should compare new treatments with established ones rather than to compare newer treatments with 'nothing'. Yet placebos are not 'nothing'. Since many new agents are toxic or ineffective, an active treatment can be worse than a placebo. The other prevailing view maintains that placebo-controlled trials are the most scientifically rigorous means of assessing efficacy of a new drug.

A major problem is that placebo effects plus spontaneous remissions can give results, which may falsely be attributed to efficacy. Independently evaluated randomised controlled trials (RCTs) are essential in trials of drugs, surgical and physical treatments. In most instances they must include a placebo component, but once greater efficacy than placebo is established, further trials are essential which compare the active (better than placebo) agent with established therapies. Benefit owing to placebo bedevils multicentre collaborative trials, which include many physicians whose personal, immeasurable therapeutic impact varies considerably.
The investigator in a placebo-controlled RCT may face a conflict between their scientific duty to follow experimental protocol, and their duty as a physician to give optimal care. ${ }^{23}$ It is plainly unethical to use a placebo if any therapy is known to be better than placebo and if withholding such therapy will compromise the patient's health.

The World Medical Association noted:

... a placebo-controlled trial may be ethically acceptable under the following circumstances: 1 . where for compelling and scientifically sound methodological reasons its use is necessary to determine the efficacy or safety of a prophylactic, diagnostic or therapeutic method; or 2 where a prophylactic, diagnostic or therapeutic method is being investigated for a minor condition and the patients who receive placebo will not be subject to any additional risk of serious or irreversible harm.

Some argue that prescribing a placebo may be unethical, akin to deceit. But it is impossible consciously to withhold placebo effects, which are often unknown to both patient and doctor. Placebos can and should be given without deception, within or outside formal trials. Further, we should not undervalue the placebo effect inherent in the doctor-patient contract, which underlies the success of some scientifically unproven treatments.

\section{Conclusions}

The placebo response is a normal human attribute and cannot be linked to age, race or type of illness. Placebos objectively affect physiological and pathological processes. Although the mechanisms are unclear, cultural factors significantly influence the frequency and susceptibility to placebo reactions. Placebos depend on expectation or conditioned belief that treatment is effective. They often operate without deliberate intention. Although imaging studies provide evidence of patterns of neural changes associated with placebo administration, the placebo mechanisms demand further scientific investigation. Controversies should not, however, prevent us from ethically deploying the placebo effect in the individual and pursuing trials when patients who receive placebo will not be harmed and provisions made to minimise risks associated with placebo.

To decide what constitutes evidence for the placebo effect, initially, there must be an additional no-treatment group to determine whether observed changes are due to the placebo. Unless accompanied by objective measurements to avoid bias, selfreported measures are unacceptable.

\section{References}

1 Pearce JMS. The placebo enigma. Q J Med 1995;88:215-20.

2 Quincy J. Quincy's Lexicon-medicum. A new medical dictionary. Revised by R Hooper. London, 1811.

3 Evans W, Hoyle C. The comparative value of drugs used in the continuous treatment of angina pectoris. Q J Med 1933;26:311-38

4 Fulda S, Wetter TC. Where dopamine meets opioids: a meta-analysis of the placebo effect in restless legs syndrome treatment studies. Brain 2008;131:902-17. 
5 Hróbjartsson A, Gøtzsche PC. Is the placebo powerless? An analysis of clinical trials comparing placebo with no treatment. $N$ Engl J Med 2001;344:1594-602.

6 Wall PD. Cited by Lowry F. Placebo response requires more study, researchers say. Can Med Assoc J 1993;148:2187-8.

7 Beecher HK. The powerful placebo. JAMA 1955;159:1602-6.

8 Cho HJ, Hotopf M, Wessely S. The placebo response in the treatment of chronic fatigue syndrome: a systematic review and meta-analysis. Psychosom Med 2005;67:301-13.

9 Bouckoms A, Hackett TP. The pain patient. In: NH Cassem (ed.), Massachusetts General Hospital handbook of general hospital psychiatry, 3rd edn. St Lois: Mosby, 1991:44-5.

10 Günther R, Herold M, Halberg E, Halberg F. Circadian placebo and ACTH effects on urinary cortisol in arthritics. Peptides 1980;1:387-90.

11 Dimond EG, Kittle CF, Crockett JE. Comparison of internal mammary artery ligation and sham operation for angina pectoris. Am J Cardiol 1960;5:483-6.

12 de Craen AJM Roos PJ, de Vries AL, Kleijnen J. Effect of colour of drugs: systematic review of perceived effect of drugs and of their effectiveness. BMJ 1996;313:1624-6.

13 Bourne HR. The rational use of placebo In: Melmon Kl, Morelli HF, Clinical pharmacology, 2nd edn. Macmillan: New York, 1978.

14 Fernandes R, Ferreira JF, Sampaio. The placebo response in studies of acute migraine. J Pediatr 2008;152:527-33.

15 Benedetti F, Lanotte M, Colloca L et al. Electrophysiological properties of thalamic, subthalamic and nigral neurons during the antiparkinsonian placebo response. J Physiol 2009;587:3869-83.
16 Hashish I, Harvey W, Harris M. Anti-inflammatory effects of ultrasound therapy: Evidence for a major placebo effect. Brit J Rheumatol 1986;25:77-81.

17 Rosenzweig P, Brohier S, Zipfel A. The placebo effect in healthy volunteers: influence of experimental conditions on the adverse events profile during phase I studies. Clin Pharmacol Ther 1993;54: $578-83$.

18 Leber P. The use of placebo control groups in the assessment of psychiatric drugs: an historical context. Bio Psych 2000;47:699-706.

19 Zubieta JK, Stohler CS. Neurobiological mechanisms of placebo responses. Ann New York Acad Sci 2009;1156:198-210.

20 Faria V, Fredrikson M, Furmark T. Imaging the placebo response: a neurofunctional review. European Neuropsychopharmacol 2008;18:473-85.

21 Voudouris NJ, Peck C, Coleman G. Conditioned response of the placebo phenomena: further support. Pain 1989;38:109-16.

22 Royal College of Physicians. Guidelines on the practice of ethics committees participants, 4th edn. London: RCP, 2007:48-50.

23 Resnik DB. The clinical investigator-subject relationship: a contextual approach. Philo Ethics Hum Med 2009;4:16.

Address for correspondence: Dr JMS Pearce, 304 Beverley Road, Anlaby, East Yorks HU10 7BG. Email: jms.pearce@me.com

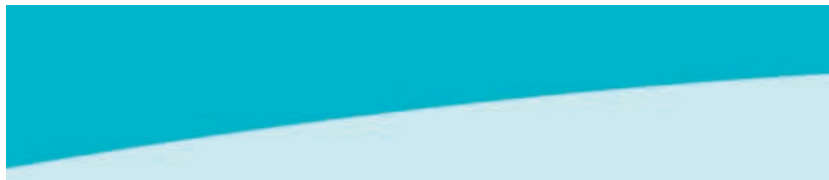

RCP accomodation

\section{William Harvey House opens}

The RCP will open the doors to William Harvey House for fellows and members on Saturday 1 October 2011. With its six large Parkside rooms, the spacious Albany Street Standard double or twin rooms and the cosy third-floor Studio rooms, William Harvey House offers comfortable and affordable accommodation in the heart of London.

The rooms, which are named after eminent physicians, retain their original character, enhanced with modern amenities and contemporary bathrooms. William Harvey House provides a 'home away from home' atmosphere for RCP fellows and members while on business or family holiday in London.

\section{Rates}

Rates range from $£ 80$ to $£ 135$ for one person ( $£ 25$ per additional guest), including $20 \%$ VAT and a cooked (weekdays) or continental (weekends) breakfast.

\section{Viewing day}

A viewing day of William Harvey House will be held on Tuesday 18 October 2011 from 1pm-4pm, with most rooms open to visitors. Please contact the William Harvey House team if you wish to attend.

\section{How to book}

To book accommodation from 1 October 2011 , please contact the William Harvey House team (details below) to make a reservation.

Tel: $+44(0) 2030751425$ Online: www.rcplondon.ac.uk/benefits/william-harvey-house Email: harveyhouse@rcplondon.ac.uk 